\title{
Use of alternative strategies by brain-damaged cats
}

\author{
FRANCIS B. COLAVITA \\ University of Pittsburgh, Pittsburgh, Pennsylvania 15260
}

\begin{abstract}
Adult cats with bilateral lesions of insular-temporal cortex were severely impaired in performing a temporal pattern discrimination. Animals prepared with such lesions at 6,5 , or 4 months of age showed progressively smaller deficits. These data initially appear to represent the standard age-related "recovery of function" phenomenon. However, a comparison of preoperative performance in cats of different ages revealed that the younger cats actually learn faster than the older ones. The data are interpreted as suggesting that some instances of age-related recovery of function following cortical damage may be due to the early-lesioned animals adopting problem solving strategies other than those utilized by adult animals and other than those intended by the experimenter.
\end{abstract}

There is a widely held belief among biological scientists that cortical damage in an immature organism is less disruptive to sensory and/or motor capacities tested in adulthood than when the same degree of injury occurs in a mature organism. The mechanism underlying this recovery of function following early cortical damage is thought to involve other brain regions "taking over" the functions of the damaged area. The neuronal plasticity underlying this takeover is thought to be uncharacteristic of the mature brain.

Several years ago we reported that kittens prepared with bilateral insular-temporal cortical lesions at 3 weeks of age showed no deficit on a temporal pattern discrimination task measured at 1 year of age (Colavita, 1972b). Adult animals prepared with similar lesions showed a profound and apparently irreversible deficit in visual (Colavita, 1972a), auditory (Colavita, 1977), and vibrotactile (Colavita, 1974) temporal pattern discriminations of the general form A-B-A vs. B-A-B. Not only did our early-lesioned animals acquire the discrimination, but also they required only half the number of trials needed by normal adult cats on a difficult visual temporal pattern discrimination task.

At the time, we interpreted these data as a striking demonstration of the standard recovery of function phenomenon. However, more recent evidence has caused us to reconsider this interpretation. We have now found (Colavita, Szeligo, \& Zimmer, 1974) that adultlesioned cats that cannot discriminate A-B-A from B-A-B can still perceive a change in the order of two successive stimuli (A-B vs. B-A), and they can still perceive a change in the repetition rate of a single stimulus (A- -A vs. -A-). This means that the whole temporal pattern discrimination (A-B-A vs. B-A-B) is equal to more than the sum of its parts (A-B vs. B-A and A- -A vs. -A-) and that adult-lesioned cats could actually solve the temporal pattern discrimination if they attended to a subset of the total stimulus array and treated the task as an ordering or repetition rate problem. In fact, they could actually learn the A-B-A vs. B-A-B discrimination faster by adopting an ordering or repetition rate strat. egy, in that ordering and repetition rate problems are learned significantly faster than the full pattern task by unoperated cats.

Based upon these data, we felt that our animals prepared with lesions at 3 weeks and tested on the temporal pattern discrimination at 1 year of age may have treated the task as an ordering or repetition rate problem. This would account for the incredibly rapid rate of acquisition shown by the animals.

Remaining to be answered was the question of why the early-lesioned animals were able to adopt an alternative problem solving strategy when the adult-lesioned animals were apparently unable to do so. The developmental literature was of some help in this regard. The insular-temporal cortex is in an undeveloped state in a 3-week-old kitten. In fact, electrocortical indices suggest that cortical development is still in progress until the kitten is 117 days of age (Scheibel \& Scheibel, 1964). Thus, animals prepared with insular-temporal lesions at 3 weeks of age should have no difficulty adopting an ordering or repetition rate strategy, in that the full pattern strategy was never available to them. Insulartemporal cortex had been ablated bilaterally before it had attained functional significance. These animals may have given the appearance of recovery of function by adopting a strategy other than the one utilized by adultlesioned cats.

This line of reasoning suggests that different ways of perceiving the environment become available to organisms with age, as neural structures continue to mature and develop. It also suggests that less complex alternative solutions may sometimes be arrived at by organisms sustaining early brain damage, giving the appearance of recovery when the "recovered" animal actually is not employing the same strategy as that employed by normal adult animals.

The present experiment was conducted to test the 
idea that, because they perceive the world differently, kittens and adult cats utilize different strategies to acquire a temporal pattern discrimination of the type studied in our earlier work. More specifically, due to their lack of cortical development, we believe that kittens must adopt an ordering or repetition rate strategy, neither of which appears to depend upon the insular-temporal region. If our hypothesis has merit, kittens prepared with insular-temporal lesions should show greater "recovery" than adult-operated cats when tested at maturity, but kittens that are old enough to undergo preoperative behavioral training should actually show faster acquisition of a temporal pattern discrimination than normal adult animals. The kitten, with its lack of cortical development, would be unable to utilize a full pattern strategy and would thus adopt an ordering or repetition rate strategy.

\section{METHOD}

\section{Subjects}

The subjects were six kittens born in our cat colony at the University of Pittsburgh. Two animals underwent preoperative behavioral training at 3 months of age and were prepared with bilateral insular-temporal lesions at 4 months; two animals were trained at 4 months of age and prepared with lesions at 5 months; the remaining two animals were trained at 5 months and prepared with lesions at 6 months.

\section{Behavioral Training}

All animals were trained in a double-grill-box shock avoidance situation; the animals made a crossing response when a change occurred in the temporal pattern of ongoing triads of $800-\mathrm{Hz}$ tone pulses at two different intensities $(82$ and $62 \mathrm{~dB}$, SPL). The recurring loud-soft-loud sequence served as the neutral signal, while a change to the soft-loud-soft temporal sequence was used as the avoidance signal. All animals were given 20 trials per day and were trained to a criterion of at least 18 out of 20 correct detections on each of 2 successive days.

Each tone pulse was $.9 \mathrm{sec}$ in duraticn. There was a .1-sec interpulse interval within a triad and a 2-sec silent interval between successive triads. On each trial the neutral signal was presented for an interval of time ranging from 45 to $120 \mathrm{sec}$. Then the avoidance signal was introduced for $20 \mathrm{sec}$. If the animal failed to make a crossing response during presentation of the avoidance signal, footshock was delivered through the floor bars of the double-grill box until an escape response was made. The shock intensity used was determined individually for each cat and consisted of the lowest level that would quickly and reliably drive the animal from one compartment of the box to the other. Crossing responses made in the absence of the avoidance signal were also punished with footshock. By the end of preoperative training the animals were no longer making spontaneous crossing responses.

\section{Surgery and Postoperative Testing}

Upon reaching criterion on the auditory-intensity temporal pattern discrimination, all six cats were prepared with bilateral lesions of insular-temporal cortex. Lesions were produced by subpial aspiration, with routine sterile precautions being observed. Antibiotics were administered postoperatively. Two weeks after surgery, the animals were retrained on the temporal pattern discrimination. Retraining was continued until either the $90 \%$ avoidance criterion was again achieved or the animal had received at least as many trials as had been required pre- operatively and there was no sign of further increments in performance. At the conclusion of postoperative testing, each animal was deeply anesthetized and perfused with saline and $10 \%$ Formalin. The brains were removed and stored in $10 \%$ Formalin prior to being sectioned on a freezing microtome at 50 microns. Serial sections through the thalamus were stained with thionin and examined for retrograde degeneration.

\section{RESULTS}

\section{Anatomical Results}

In all six animals the ventral boundary of the ablations extended to the rhinal fissure. The resulting pattern of retrograde degeneration in the thalamus was similar to that reported in our previous studies.

\section{Behavioral Results}

The mean number of preoperative trials to criterion for 3-, 4-, and 5-month-old kittens is shown in Table 1. Also included in the table for comparison purposes is a mean value obtained in our earlier work on auditory temporal pattern discrimination with normal adult cats.

The amount of postoperative recovery on the temporal pattern discrimination task demonstrated by pairs of kittens prepared with bilateral insular-temporal lesions at 4,5 , or 6 months of age is presented in Table 2. Also included in the table is the mean recovery value seen in our earlier work with adult-lesioned animals.

\section{DISCUSSION}

The data summarized in Table 2 suggest that the younger the animal at the time of insular-temporal ablation, the higher the level of postoperative performance on the temporal pattern discrimination task. Thus, while the typical recovery level seen in adult-operated cats is $10 \%$, the recovery level achieved by our animals lesioned at 4 months was $90 \%$.

At first glance these data appear to represent another instance of the standard recovery of function finding, where undamaged brain regions in the young animal are thought to take over the function of the damaged areas to a greater extent than in the older animal. However, when one considers the preoperative behavioral data depicted in Table 1 , an alternative

Table 1

Mean Preoperative Trials to Criterion for Animals Trained at Different Ages

\begin{tabular}{cclc}
\hline Age & N of Trials & \multicolumn{1}{c}{ Age } & N of Trials \\
\hline 3 Months & 180 & 5 Months & 290 \\
4 Months & 270 & Adult & 320 \\
\hline
\end{tabular}

Table 2

Postoperative Recovery for Animals Prepared With Bilateral Lesions at Different Ages

\begin{tabular}{cccc}
\hline Age & $\begin{array}{c}\text { Percent } \\
\text { Recovery }\end{array}$ & \multicolumn{1}{c}{ Age } & $\begin{array}{c}\text { Percent } \\
\text { Recovery }\end{array}$ \\
\hline 4 Months & 90 & 6 Months & 45 \\
5 Months & 65 & Adult & 10 \\
\hline
\end{tabular}


explanation for the age-related differences in postoperative performance gains credibility. Table 1 shows a clear trend for animals to require more preoperative trials to reach the $90 \%$ performance criterion as they increase in age from 3 months to adulthood. These data suggest to us that the younger animals were probably utilizing an ordering or repetition rate strategy, the older animals were probably utilizing a strategy based upon attention to the entire stimulus configuration (what we have referred to in earlier work as the full pattern strategy), and the 4- and 5-month-old animals may have been utilizing some combination of both types of strategies. What appears to be a typical recovery of function relationship in Table 2 may actually be a reflection of the degree to which insular-temporal cortex was involved in original learning. If original learning occurred at a time when insular-temporal cortex was in an undeveloped state, then the animal had to utilize a problem solving strategy that did not depend upon this cortical area. Such an animal would show little or no behavioral disruption on a temporal pattern discrimination task following bilateral insular-temporal cortical ablation. In animals where insular-temporal cortex had attained a higher stage of development at the time of original learning, some postoperative disruption of performance should be evident; in animals where insular-temporal cortex had attained full maturity at the time of original learning, a severe postoperative learning deficit should be apparent.

To briefly summarize, our data suggest that some instances of recovery of function may actually be the result of an animal that has sustained early brain damage adopting a strategy other than the one intended by the experimenter, other than the one utilized by normal adult animals, and other than the one utilized (unsuccessfully) by animals sustaining cortical damage at maturity. This is not to say, of course, that all demonstrations of recovery of function can be accounted for in this manner. However, we would advise caution in the interpretation of recovery of function data in which the performance of the "recovered" animals appears to exceed that of normal controls. There are several such instances in the recovery literature.

\section{REFERENCES}

Colavita, F. B. Auditory cortical lesions and visual pattern discrimination in cat. Brain Research, 1972, 39, 437-447. (a)

Colavita, F. B. Recovery of function following insular-temporal lesions in kittens. T.I.T. Journal of Life Sciences, 1972, 2, 101-105. (b)

Colavita, F. B. Insular-temporal lesions and vibrotactile temporal pattern discrimination in cats. Physiology and Behavior, 1974, 12, 215-218.

Colavita, F. B. Theoretical review: Temporal pattern discrimination in the cat. Physiology and Behavior, 1977, 18, 513-521.

Colavita, F. B., Szeligo, F. V., \& Zimmer, S. D. Temporal pattern discrimination in cats with insular-temporal lesions. Brain Research, 1974, 79, 153-156.

Scheibel, M., \& Scheibel, A. Some structural and functional substrates of development in young cats. In W. Himwich \& H. Himwich (Eds.), The developing brain. New York: Elsevier, 1964.

(Received for publication November 19, 1979.) 